\title{
HNF-4 $\alpha$ promotes multidrug resistance of gastric cancer cells through the modulation of cell apoptosis
}

\author{
YUBO MA ${ }^{1}, \mathrm{XUFU}_{\mathrm{WEI}^{2} \text { and ZHONGJUN WU }}{ }^{2}$ \\ ${ }^{1}$ The First Clinical College; ${ }^{2}$ Department of Hepatobiliary Surgery, The First Affiliated Hospital, \\ Chongqing Medical University, Chongqing 400016, P.R. China
}

Received September 30, 2016; Accepted August 17, 2017

DOI: $10.3892 / 01.2017 .7095$

\begin{abstract}
Multidrug resistance (MDR) typically leads to treatment failure, and is associated with disease progression of gastric cancer (GC). In the present study, a total of 15 aberrantly activated transcription factors (TFs) were detected in chemo-resistant GC cells using a TF Activation Profiling Plate Array. Among these TFs, hepatocyte nuclear factor (HNF) $-4 \alpha$ was significantly upregulated in multidrug-resistant GC cells $(\mathrm{P}=0.019)$. The overexpression of $\mathrm{HNF}-4 \alpha$ was able to cause resistance to multiple chemotherapeutics, whereas inhibition of HNF- $4 \alpha$ appeared to reverse cancer cell resistance. Further studies demonstrated that HNF- $4 \alpha$ had no clear influence on drug transportation; however, inhibition of drug-induced cell apoptosis occurred as B-cell lymphoma 2 (Bcl-2) expression increased in GC cells. Additionally, immunohistochemistry demonstrated that HNF-4 $\alpha$ was overexpressed in human GC tissues, and associated with tumor stage and lymph node metastasis. In conclusion, the results of the present study indicate the involvement of TFs in MDR in GC, and suggest that HNF- $4 \alpha$ may enhance MDR in GC by regulating cell apoptosis and $\mathrm{Bcl}-2$ expression.
\end{abstract}

\section{Introduction}

Gastric cancer (GC) is one of the most common malignant tumors worldwide, particularly in China and other Asian countries (1). Chemotherapy is the standard strategy used

Correspondence to: Dr Zhongjun Wu, Department of Hepatobiliary Surgery, The First Affiliated Hospital, Chongqing Medical University, 1 Youyi Road, Chongqing 400016, P.R. China E-mail:wzjtcy@126.com

Abbreviations: GC, gastric cancer; HIF, hypoxia-inducible factor; $\mathrm{HNF}$, hepatocyte nuclear factor; IDH, isocitrate dehydrogenase; IHCC, intrahepatic cholangiocarcinoma; MDR, multidrug

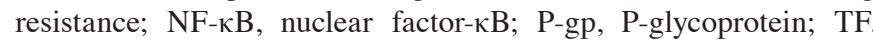
transcription factor

Key words: apoptosis, B-cell lymphoma 2, gastric cancer, hepatocyte nuclear factor- $4 \alpha$, multidrug resistance, transcription factor to manage $\mathrm{GC}$; however, the majority of patients fail to achieve the ideal initial response and/or develop resistance to chemotherapy. Multidrug resistance (MDR) is one of the primary mechanisms for failure of GC treatment. A previous study demonstrated that aberrant drug transportation and cell apoptosis contributed to chemotherapeutic resistance as drug transporters were demonstrated to be an essential component in intracellular drug metabolism (2). P-glycoprotein (P-gp) is one of the most studied transporters in drug resistance. A large population of chemotherapeutic drugs are substrates for $\mathrm{P}-\mathrm{gp}$, and thus expression or function of P-gp was associated with MDR in several types of cancer $(2,3)$. Certain types of cancer cells, including breast cancer, head and neck squamous cell carcinoma and colorectal cancer cells are able to activate several signaling transduction pathways in response to drug stimuli, and also alter the expression and protein activity of apoptosis-associated molecules in order to resist the action of therapy and allow cancer cells to survive $(2,4)$. Currently, multiple molecules, including glutathione $\mathrm{S}$-transferase, breast cancer resistance protein, PI3K/Akt, Bcl-2 and p53 have been proved to be associated with MDR in chemotherapy (2-4). However, the underlying molecular mechanism and associated molecular interactions remain unclear.

Transcription factors (TFs) are able to target promoter regions of different genes, leading to the regulation of a large group of target genes. Previous studies have identified that TFs serve multiple critical roles in cancer MDR. The inactivation or mutation of p53, a well-known master tumor suppressor, was reported to induce drug resistance through the modulation of apoptosis-associated proteins (5-7). Nuclear factor- $\kappa \mathrm{B}(\mathrm{NF}-\kappa \mathrm{B})$ was also revealed to promote MDR by targeting apoptosis-associated molecules or microRNAs (8-10). Furthermore, NF- $\kappa \mathrm{B}$ may also possess a compensatory function with p53 through mutual interaction and thereby regulate the response to 5-fluorouracil treatment (8). As well as the classical association with drug response, there is also a series of MDR-associated TFs that have been identified. In drug-resistant GC cells, the expression of zinc ribbon domain-containing 1 (ZNRD1) is increased, and therefore inhibition of ZNRD1may be able to improve drug sensitivity to various chemotherapeutics (11). A previous study also reported that a significant decrease in cut-like homeobox 1 (CUTL1) transcriptional activity may participate in doxorubicin resistance (12). Therefore, targeting MDR-associated TFs may be an efficient strategy in MDR reversion. 
In the present study, a high-throughput TF activation-profiling assay was utilized to analyze activities of TFs between drug-resistant and drug-sensitive GC cells. A total of 15 TFs with aberrant activities were detected. Among these TFs, few previous reports have commented on the association between hepatocyte nuclear factor (HNF)- $4 \alpha$ and MDR. Furthermore, ectopic expression of HNF- $4 \alpha$ promoting drug resistance in GC was exhibited, whereas the loss of HNF- $4 \alpha$ increased drug sensitivity in vitro. In addition, it was revealed that HNF- $4 \alpha$ was able to regulate MDR by targeting B-cell lymphoma 2 (Bcl-2), without marked effects on intracellular chemotherapeutic drug transportation.

\section{Materials and methods}

Patient characteristics of tissue specimens. In total, 126 GC tissue samples and 69 chronic gastritis (CG) tissue samples were obtained from the Pathology Department of Xijing Hospital (Shaanxi, China). GC tissue samples were from 60 males and 66 females, and were categorized on the basis of age (above or below 56 years), tumor differentiation (well, moderate and poor), tumor stage (T1-4) and lymph node metastasis (N0-N3). Corresponding clinical data were obtained from medical records and all patients with GC were followed-up for 70 months. The present study was approved by the Institutional Review Board of Xijing Hospital, Xi'an, China.

Cell culture and TF Activation Profiling Plate Array. Human GC cell lines including KATO III, AGS and immortalized human gastric epithelial cell line GES-1 were obtained from the Shanghai Institute of Biochemistry and Cell (Chinese Academy of Sciences, Shanghai, China) and maintained within the Department of Hepatobiliary Surgery, the First Affiliated Hospital (Chongqing Medical University, Chongqing, China). Human GC cell lines GC9811, SGC7901 and MDR variant SGC7901/VCR (derived from SGC7901 by stepwise selection with vincristine) were obtained from the Department of Digestive Diseases, Xijing Hospital (Shaanxi, China). All cells were cultured in RPMI-1640 medium (Hyclone; GE Healthcare, Logan, UT, USA) containing 10\% fetal bovine serum (Gibco; Thermo Fisher Scientific, Inc., Waltham, MA, USA) and $1 \%$ penicillin/streptomycin and maintained at $37^{\circ} \mathrm{C}$ under an atmosphere of $5 \% \mathrm{CO}_{2}$. The nucleoprotein extracts of SGC7901 and SGC7901/VCR cells were prepared and subjected to TF Activation Profiling Plate Array (Signosis, Inc., Santa Clara, CA, USA), according to the manufacturer's protocol. The TF Activation Profiling Plate Array was used to determine the activities of 96 TFs in one plate. The activity of each TF was automatically recorded and 1.5 was set as the threshold value for screening over-activated TFs.

Lentiviral infection and stable cell variants. Lentiviruses expressing HNF-4 $\alpha$, specific small interfering RNA (siRNA) or the corresponding controls were products from Shanghai Genechem Co., Ltd. (Shanghai, China). Target cells were infected with lentiviruses according to the manufacturer's protocol and mixed stable clones were isolated and subjected to in vitro drug sensitivity assay, apoptosis assay, intracellular adriamycin concentration analysis and western blotting.
In vitro drug sensitivity assay. Drug sensitivity was evaluated in vitro using an MTT assay (Merck KGaA, Darmstadt, Germany) as described previously (11). Briefly, cells $\left(5 \times 10^{3}\right)$ were seeded into 96 -well plates and incubated at $37^{\circ} \mathrm{C}$ with Adriamycin, 5-fluorouracil, cisplatin, vincristine and mitomycin for $48 \mathrm{~h}$ at $0.01-, 0.1$-, 1- and 10-fold peak concentration in human sera. Peak concentrations for Adriamycin, 5-fluorouracil, cisplatin, vincristine and mitomycin were $0.4,10.0,3.0$, 0.5 and $3.0 \mu \mathrm{g} / \mathrm{ml}$ respectively. MTT was added to the wells and the optical density at wave length $570 \mathrm{~nm}$ was measured $4 \mathrm{~h}$ later. The inhibition rates and half-maximal inhibitory concentration $\left(\mathrm{IC}_{50}\right)$ values were then calculated.

Apoptosis assay. GC SGC7901 cells and variants overexpressing $\mathrm{HNF}-4 \alpha$ were treated with $0.25 \mu \mathrm{g} / \mathrm{ml}$ vincristine. SGC7901/VCR cells and their variants with knockdown of HNF-4 $\alpha$ were treated with $2.5 \mu \mathrm{g} / \mathrm{ml}$ vincristine. Following incubation at $37^{\circ} \mathrm{C}$ for $24 \mathrm{~h}$ with vincristine, the apoptotic cells were analyzed using flow cytometry using an Annexin V-fluorescein isothiocyanate (FITC) apoptosis detection kit (BD Biosciences, Franklin Lakes, NJ, USA) as described previously (11). Briefly, cell samples were sequentially incubated with Annexin V-fluorescein isothiocyanate and propidium iodide (PI) following the manufacturer's protocol and then analyzed with a flow cytometer (FACSCalibur; BD Biosciences, San Jose, CA, USA) using a 530/30 nm signal detector for Annexin V-FITC and a 582/42 nm signal detector for PI. The data were subsequently analyzed by Flow J software (version 7.6.5; Tree Star, Inc., San Carlos, CA, USA). The upper left and lower left quadrants represented late and early apoptosis, respectively. The total apoptosis ratio was calculated by adding the late and early apoptosis proportions.

Intracellular Adriamycin concentration analysis. The intracellular accumulation and retention of Adriamycin was determined using flow cytometry. GC cells and their variants were inoculated into 6 -well plates and allowed to adhere overnight at $37^{\circ} \mathrm{C}$. Adriamycin $(5 \mathrm{mg} / \mathrm{ml})$ was added and cells were incubated at $37^{\circ} \mathrm{C}$ in Adriamycin-containing RPMI-1640 medium with $10 \%$ fetal bovine serum for $1 \mathrm{~h}$. To detect Adriamycin retention, cells were transferred to Adriamycin-free RPMI-1640 medium with $10 \%$ fetal bovine serum for another $1 \mathrm{~h}$ and then trypsinized, washed, resuspended in phosphate buffered saline (PBS) and subjected to flow cytometry. A flow cytometer (FACSCalibur; BD Biosciences, San Jose, CA, USA) was used with a 582/42 nm signal detector for intracellular Adriamycin. The data were subsequently analyzed by Flow J software (version 7.6.5; Tree Star, Inc.). Mean fluorescence intensity of Adriamycin was obtained and expressed as the mean \pm standard error of the mean. The Adriamycin-releasing index was calculated as $100 \% \mathrm{x}$ (mean fluorescence intensity of accumulation-mean fluorescence intensity of retention)/(mean fluorescence intensity of accumulation). Experiments were performed in triplicate.

Western blotting. Cells were lysed in radioimmunoprecipitation buffer (Beyotime Institute of Biotechnology, Haimen, China) supplemented with $1 \mathrm{mM}$ phenylmethylsulfonyl fluoride and $10 \mu \mathrm{g} / \mathrm{ml}$ each of pepstatin $\mathrm{A}$, leupeptin, chymostatin 
and aprotinin (Roche Diagnostics, Basel, Switzerland). Protein concentration was measured with a Bicinchoninic acid Protein Assay kit according to the manufacturer's protocol (Thermo Scientific Pierce, Rockford, IL, USA). Western blots were performed according to standard methods as described previously (8). Equal amounts of protein $(50 \mu \mathrm{g})$ were loaded onto a SDS-PAGE gel (8-12\% polyacrylamide) and subjected to electrophoresis at $200 \mathrm{~V}$ for $50 \mathrm{~min}$, transferred to nitrocellulose and blocked overnight at $4^{\circ} \mathrm{C}$ in blocking buffer $(\mathrm{NaCl}$ $250 \mathrm{mmol} / 1,0.02 \%$ Tween $20,5 \%$ goat serum and $3 \%$ bovine serum albumin). Primary antibodies were added for $3 \mathrm{~h}$ at room temperature. Blots were washed, and species-matched peroxidase-conjugated secondary antibody was added (1:2,000). Labeled bands from washed blots were detected using an enhanced chemiluminescence kit (Amersham, Louisville, CO, USA). Primary antibodies against HNF- $4 \alpha$ (1:1,000; cat. no. 3113; Cell Signaling Technology, Inc., Danvers, MA, USA), Bcl-2-associated X protein (Bax; 1:500; cat. no. sc-6236; Santa Cruz Biotechnology, Inc., Dallas, TX, USA), Bcl-2 homologous antagonist killer (Bak; 1:500; cat. no. sc-832, Santa Cruz Biotechnology, Inc.), B-cell lymphoma extra-large (Bcl-xL; 1:500; cat. no. sc-7195, Santa Cruz Biotechnology, Inc.), caspase-3 (1:1,000; cat. no. 9662, Cell Signaling Technology, Inc.), cleaved caspase-3 (1:1,000; cat. no. 9661, Cell Signaling Technology, Inc.), Bcl-2 (1:500; cat. no. 04-436, Merck KGaA, Darmstadt, Germany) and $\beta$-actin (1:2,000; cat. no. MABT825, Merck KGaA) were used. The secondary antibodies included horseradish peroxidase (HRP)-conjugated anti-rabbit immunoglobulin (Ig)G (1:2,000; cat. no. 7074, Cell Signaling Technology, Inc.) and HRP-conjugated anti-mouse IgG (1:3,000; cat. no. 7076, Cell Signaling Technology, Inc.).

Tissue specimens and immunohistochemistry. Immunohistochemical examination was performed using the streptavidin-biotin complex method. Fresh gastric tissues were fixed in $4 \%$ formalin overnight at room temperature and embedded in paraffin. The tissue blocks were cut into sections (4 $\mu \mathrm{m}$ thick). Prior to staining, the sections were treated with $0.3 \%$ hydrogen peroxide in $100 \%$ methanol for $30 \mathrm{~min}$ at room temperature and then washed in PBS. Following incubation with normal goat serum for $10 \mathrm{~min}$, the sections were incubated with anti-HNF-4 $\alpha$ (1:200; cat. no. 3113, Cell Signaling Technology, Inc.) overnight at $4^{\circ} \mathrm{C}$. Afterincubation, the sections were incubated at room temperature for $1 \mathrm{~h}$. They were washed twice in PBS and treated with biotinylated goat anti-rabbit IgG (1:100; cat. no. SA2002, Boster, Wuhan, China) and peroxidase-conjugated streptavidin (1:100; cat. no. SA2002, Boster) for $30 \mathrm{~min}$ at room temperature. They were then reacted with $0.02 \%$ diaminobenzidine tetrahydrochloride containing $0.005 \%$ hydrogen peroxide for 4 min and counterstained with hematoxylin. Rabbit normal serum was used to replace the primary antibody as a blank control. The stained sections were observed using a light microscope (magnification, x200). The expression of $\mathrm{HNF}-4 \alpha$ was evaluated according to the ratio of positive cells per specimen (R) and staining intensity (I) as described previously (13). A total score (RxI) of 0 to 12 was calculated and graded as follows: negative (-, 0 to 2$)$, weak positive (,+ 3 to 5$)$, moderate positive (,++ 6 to 9$)$ and strong positive (+++, 10 to 12$)$.
Table I. Screening for aberrantly activated TFs in chemo-resistant GC cells.

\begin{tabular}{|c|c|c|}
\hline $\begin{array}{l}\text { Transcription } \\
\text { factor }\end{array}$ & $\begin{array}{c}\text { Activity ratio } \\
(\text { VCR/SGC7901) }\end{array}$ & P-value \\
\hline $\mathrm{NF}-\kappa \mathrm{B}$ & 2.17 & 0.006 \\
\hline HIF-1 & 1.95 & 0.026 \\
\hline HNF-4 $4 \alpha$ & 1.92 & 0.019 \\
\hline RXR & 1.89 & 0.025 \\
\hline GATA & 1.87 & 0.021 \\
\hline STAT1 & 1.74 & 0.031 \\
\hline STAT3 & 1.69 & 0.024 \\
\hline 4-Oct & 1.68 & 0.017 \\
\hline C/EBP & 1.64 & 0.004 \\
\hline KLF4 & 1.58 & 0.011 \\
\hline Snail & 1.56 & 0.028 \\
\hline NFAT & 1.55 & 0.035 \\
\hline TCF/LEF & 1.54 & 0.007 \\
\hline AP1 & 1.52 & 0.009 \\
\hline ATF2 & 1.5 & 0.016 \\
\hline
\end{tabular}

Human GC cell line SGC7901 and its multidrug-resistant variant SGC7901/VCR were used to screen for TFs associated with chemo-resistance. The TF Activation Profiling Plate Array was employed to determine activities of $96 \mathrm{TFs}$ in one plate. The activity of each TF was automatically recorded and 1.5 was set as the threshold value for screening over-activated TFs. GC, gastric cancer; TF, transcription factor; NF- $\mathrm{\kappa B}$, nuclear factor- $\mathrm{kB}$; HIF-1, hypoxia-inducible factor 1 ; HNF- $4 \alpha$, hepatocyte nuclear factor $4 \alpha$; RXR, retinoid X receptor; GATA, GATA-binding protein; STAT, signal transducer and activator of transcription; 4-Oct, octamer-binding transcription factor 4; C/EBP, CCAAT/enhancer-binding protein; KLF4, Krüppel-like factor 4; NFAT, nuclear factor of activated T-cells; $\mathrm{TCF} / \mathrm{LEF}$, transcription factor/lymphoid enhancer-binding factor 1 ; AP1, activating protein-1; ATF2, activating transcription factor 2 .

Statistical analysis. SPSS software (version 17.0; SPSS, Inc., Chicago, IL, USA) was used to perform statistical analysis. One-way analysis of variance or two-tailed unpaired Student's t-test was used to analyze the data of TF activity, $\mathrm{IC}_{50}$ values, cell apoptosis and intracellular Adriamycin. A $\chi^{2}$ test was applied to detect the significance of the difference in HNF- $4 \alpha$ expression frequency in human gastric tissues and its clinicopathological association in GC. Kaplan-Meier estimator survival curves were created to analyze the association of HNF-4 $\alpha$ with patient survival, and the log-rank test was used to compare the difference of survival curves among groups. $\mathrm{P}<0.05$ was considered to indicate a statistically significant difference.

\section{Results}

Identification of MDR-associated TFs by TF Activation Profiling Plate Array. To identify TFs involved in MDR in GC, nucleoproteins were extracted from chemo-sensitive (SGC7901) and chemo-resistant GC cells (SGC7901/VCR) for TF Activation Profiling Plate Array. Results presented in Table I 
Table II. Effects of HNF-4 $\alpha$ on drug sensitivities of GC cells.

\begin{tabular}{lccccc}
\hline & \multicolumn{5}{c}{$\mathrm{IC}_{50}$ values, $\mu \mathrm{g} / \mathrm{ml}$} \\
\cline { 2 - 6 } Cell line & ADR & 5 -Fu & CDDP & VCR & MMC \\
\hline SGC7901 & $0.52 \pm 0.05$ & $3.11 \pm 0.22$ & $1.63 \pm 0.21$ & $0.25 \pm 0.01$ & $1.39 \pm 0.16$ \\
SGC7901-Ctrl & $0.61 \pm 0.11$ & $2.89 \pm 0.34$ & $1.93 \pm 0.28$ & $0.29 \pm 0.08$ & $1.56 \pm 0.23$ \\
SGC7901-HNF4 $\alpha$ & $1.58 \pm 0.29^{\mathrm{a}}$ & $6.98 \pm 0.73^{\mathrm{a}}$ & $4.16 \pm 0.52^{\mathrm{a}}$ & $1.73 \pm 0.31^{\mathrm{a}}$ & $4.55 \pm 0.67^{\mathrm{a}}$ \\
SGC7901/VCR & $5.44 \pm 0.47$ & $9.17 \pm 1.15$ & $7.65 \pm 0.94$ & $5.86 \pm 0.69$ & $8.22 \pm 1.03$ \\
SGC7901/VCR-siCtrl & $6.21 \pm 0.79$ & $10.83 \pm 1.57$ & $7.02 \pm 0.86$ & $6.29 \pm 0.88$ & $9.03 \pm 1.27$ \\
SGC7901/VCR-siHNF4 $\alpha$ & $2.95 \pm 0.38^{\mathrm{b}}$ & $8.21 \pm 0.93^{\mathrm{b}}$ & $5.14 \pm 0.78^{\mathrm{b}}$ & $3.46 \pm 0.52^{\mathrm{b}}$ & $6.11 \pm 0.94^{\mathrm{b}}$ \\
\hline
\end{tabular}

HNF4 $\alpha$ was overexpressed in SGC7901 cells and suppressed in SGC7901/VCR cells with lentiviruses expressing HNF-4 $\alpha$ or its specific small interfering RNA (siHNF4 $\alpha$ ). The sensitivity of SGC7901 and SGC7901/VCR cells, and their variants, was determined in vitro using an MTT assay. SGC7901-Ctrl and SGC7901/VCR-siCtrl cells were infected with control lentiviruses. ${ }^{a}$ P<0.01 vs. SGC7901 and SGC7901-Ctrl. ${ }^{b} \mathrm{P}<0.01$ vs. SGC7901/VCR and SGC7901/VCR-siCtrl. Results are representative of 3 independent experiments. IC $_{50}$, half-maximal inhibitory concentration; Ctrl, control; ADR, Adriamycin; 5-Fu, 5-fluorouracil; CDDP, cisplatin; VCR, vincristine; MMC, mitomycin; HNF-4 $\alpha$, hepatocyte nuclear factor- $4 \alpha$.

identify the increased activity of $15 \mathrm{TFs}$ in chemo-resistant GC cells SGC7901/VCR compared with SGC7901. Among the top three aberrantly activated TFs, NF- $\mathrm{\kappa B}$ and hypoxia-inducible factor (HIF)-1 were frequently reported to regulate drug resistance in GC, followed by HNF-4 $\alpha$, a well-known TF in hepatocyte differentiation and whose potential roles in MDR were not previously completely investigated. The western blot analysis determined the expression levels of HNF- $4 \alpha$ in multiple GC cell lines. HNF- $4 \alpha$ was increased in GC cell lines compared with immortalized gastric epithelial cells, and the expression of HNF- $4 \alpha$ was also upregulated in chemo-resistant cells (SGC7901/VCR) compared with chemo-sensitive cells (SGC7901; Fig. 1A).

$H N F-4 \alpha$ regulates MDR of GC cells in vitro. Cell models with stable lentiviral transfection for HNF- $4 \alpha$ or its siRNA were established (Fig. 1B) and drug sensitivities were measured using MTT assays (Table II). The overexpression of HNF- $4 \alpha$ markedly promoted resistance to chemotherapeutic drugs, which demonstrated an increase in the $\mathrm{IC}_{50}$ values of Adriamycin, vincristine, 5-fluorouracil, cisplatin and mitomycin. Furthermore, inhibition of HNF- $4 \alpha$ resulted in a decrease in the $\mathrm{IC}_{50}$ values of these drugs (Table II).

$H N F-4 \alpha$ has no effect on drug transport in GC cells. Enhanced drug efflux activity is one of the primary causes of drug resistance. To test whether HNF- $4 \alpha$ was able to affect drug transportation, intracellular Adriamycin assays were performed using flow cytometry. Fluorescence intensity of accumulated and retained Adriamycin was markedly decreased in SGC7901/VCR cells compared with SGC7901 cells (Fig. 2A and B), indicating that drug transportation was active in chemo-resistant GC cells. However, neither ectopic expression nor knockdown of HNF- $4 \alpha$ was able to lead to any change in Adriamycin accumulation and retention (Fig. 2A and B). Although SGC7901/VCR cells exhibited a significantly increased Adriamycin releasing index compared with SGC7901 cells $(\mathrm{P}<0.01)$, modulation of HNF-4 $\alpha$ expression displayed no influence on Adriamycin release from GC cells (Fig. 2C).

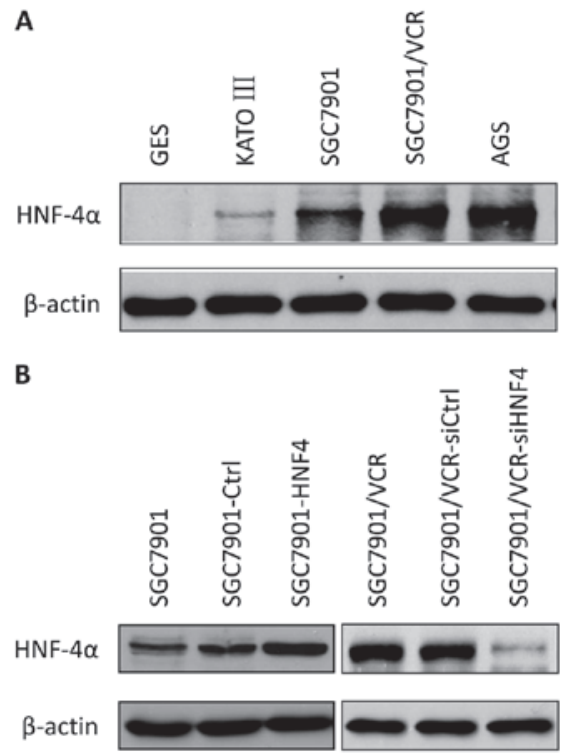

Figure 1. HNF-4 $\alpha$ is overexpressed and modulated in GC cell lines and their variants. (A) Nuclear proteins were exacted from $6 \mathrm{GC}$ cell lines and HNF-4 $\alpha$ expression levels of GC cells were compared with immortalized gastric epithelial cells using western blot analysis. (B) GC cell line SGC7901 and the multidrug-resistant strain SGC7901/VCR were stably infected with lentiviruses expressing HNF- $4 \alpha, \mathrm{HNF}-4 \alpha$-specific siRNA or their corresponding controls. Western blotting was performed to determine the expression of HNF- $4 \alpha$ in GC cell variants. $\beta$-actin served as a loading control. The results are representative of 4 independent experiments. HNF- $4 \alpha$, hepatocyte nuclear factor $4 \alpha$; VCR, vincristine; Ctrl, control; GC, gastric cancer.

HNF-4a modulates cell apoptosis through targeting Bcl-2 expression. The effects of HNF- $4 \alpha$ on vincristine-triggered cell apoptosis were evaluated in vitro. It was revealed that cell apoptosis was suppressed in SGC7901 cells with HNF-4 $\alpha$ overexpression (Fig. 3A). Conversely, increasing apoptosis in SGC7901/VCR cells was observed following HNF-4 $\alpha$ knockdown (Fig. 3A). Cleaved caspase-3 was also analyzed in vincristine-treated cells (Fig. 3B). As indicated in Fig. 3B, ectopic expression of HNF- $4 \alpha$ resulted in decreased cleaved caspase-3, whereas knockdown of HNF-4 $\alpha$ led to enhanced 
Table III. Clinicopathological association of HNF-4 $\alpha$ in human GC tissues.

\begin{tabular}{|c|c|c|c|c|c|}
\hline \multirow[b]{2}{*}{ Characteristics } & \multicolumn{4}{|c|}{ HNF-4 $\alpha$ expression } & \multirow[b]{2}{*}{ P-value } \\
\hline & - & + & ++ & +++ & \\
\hline $\mathrm{n}$ & 2 & 15 & 48 & 61 & \\
\hline Age (years) & & & & & 0.682 \\
\hline$<56$ & 0 & 8 & 23 & 32 & \\
\hline$\geq 56$ & 2 & 7 & 25 & 29 & \\
\hline Gender & & & & & 0.563 \\
\hline Male & 1 & 6 & 26 & 27 & \\
\hline Female & 1 & 9 & 22 & 34 & \\
\hline Differentiation & & & & & 0.499 \\
\hline Well & 1 & 5 & 15 & 18 & \\
\hline Moderate & 1 & 3 & 20 & 21 & \\
\hline Poor & 0 & 7 & 13 & 22 & \\
\hline Tumor stage & & & & & 0.015 \\
\hline $\mathrm{T} 1$ & 0 & 6 & 8 & 7 & \\
\hline $\mathrm{T} 2$ & 1 & 4 & 13 & 14 & \\
\hline $\mathrm{T} 3$ & 1 & 2 & 15 & 18 & \\
\hline $\mathrm{T} 4$ & 0 & 3 & 12 & 22 & \\
\hline Lymph node metastasis & & & & & 0.007 \\
\hline NO & 0 & 4 & 10 & 6 & \\
\hline $\mathrm{N} 1$ & 1 & 5 & 12 & 9 & \\
\hline $\mathrm{N} 2$ & 0 & 3 & 12 & 20 & \\
\hline N3 & 1 & 3 & 14 & 26 & \\
\hline
\end{tabular}

Expression of HNF-4 $\alpha$ was detected using immunohistochemical staining in 126 cases of human gastric cancer tissue specimens. Association of HNF-4 $\alpha$ with clinicopathological factors was evaluated using SPSS statistical software. HNF-4 $\alpha$, hepatocyte nuclear factor- $4 \alpha$

cleaved caspase-3. Detection of expression levels of the apoptosis-associated molecules Bcl-2, Bax, Bcl-xL and Bak demonstrated that the expression of $\mathrm{Bcl}-2$ was upregulated in $\mathrm{HNF}-4 \alpha$-overexpressed cells, as well as downregulated in cells with knock-down of HNF-4 $\alpha$ (Fig. 3B). Furthermore, the expression of Bax, Bcl-xL and Bak were not influenced by HNF-4 $\alpha$ (Fig. 3C and D).

$H N F-4 \alpha$ is overexpressed in human GC tissues. To elucidate the clinical relevance of HNF- $4 \alpha$ in GC, an immunohistochemical assay to determine HNF- $4 \alpha$ expression in human GC tissues was performed. ACG tissue specimen was used as a control. It was demonstrated that HNF-4 $\alpha$ was extensively expressed in GES cells, localized in both nuclei and cytoplasm (Fig. 4A-D). Staining of HNF-4 $\alpha$ was weak in CG, although it was much stronger in GC tissues. As presented in Fig. 4E, the difference in $\mathrm{HNF}-4 \alpha$ staining in GC and $\mathrm{CG}$ was statistically significant $(\mathrm{P}<0.01)$. The clinical and pathological association of HNF- $4 \alpha$ expression was further analyzed. It was demonstrated that HNF- $4 \alpha$ expression in GC tissues was associated with tumor stage and lymph node metastasis; however, it was not associated with age, sex or tumor differentiation (Table III).
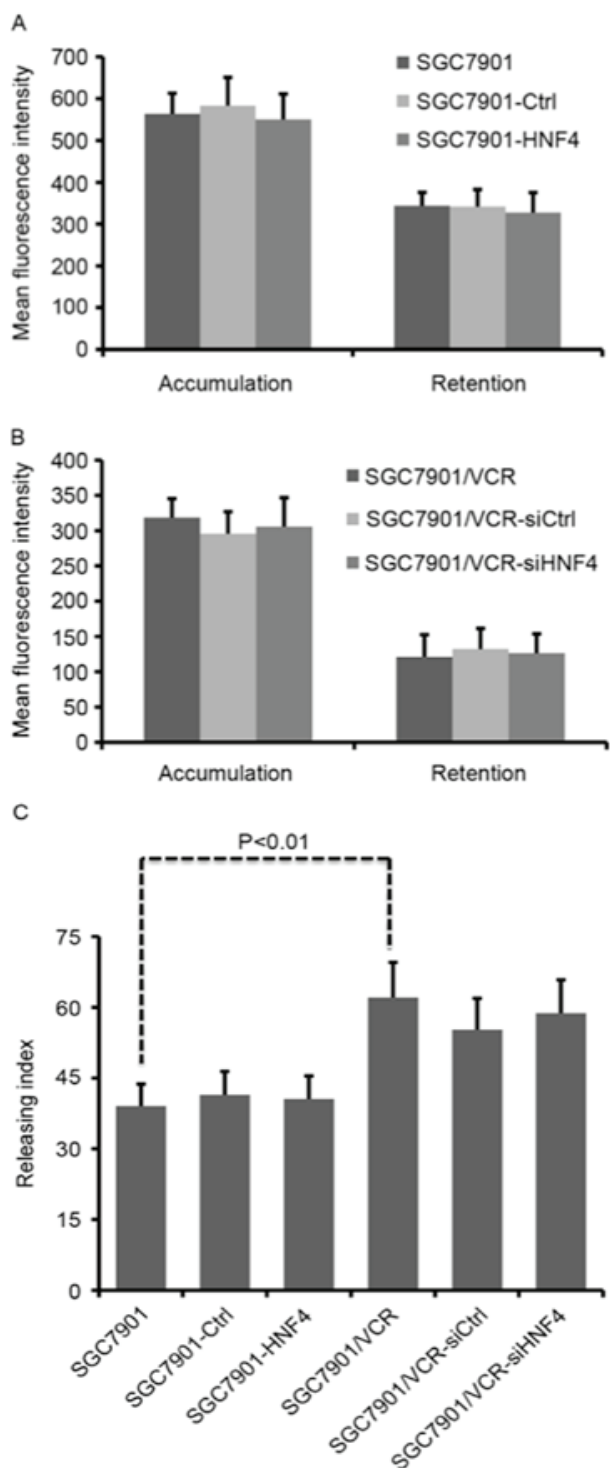

Figure 2. HNF-4 $\alpha$ has no clear influence on intracellular accumulation and retention of Adriamycin in gastric cancer cells. Gastric cancer cells and their variants were incubated with $5 \mathrm{mg} / \mathrm{ml}$ Adriamycin for $1 \mathrm{~h}$. (A) Cells were directly harvested and subjected to flow cytometry to detect Adriamycin accumulation. (B) Cells were changed to drug-free medium for another $1 \mathrm{~h}$ and then harvested to detect Adriamycin retention. Intracellular Adriamycin was determined using flow cytometry and fluorescence intensity was expressed as the mean \pm standard error of the mean. (C) The Adriamycin releasing index was calculated as: $100 \% \mathrm{x}$ (mean fluorescence intensity of accumulation-mean fluorescence intensity of retention)/(mean fluorescence intensity of accumulation). Results were from 3 independent experiments. Ctrl, control; HNF4, hepatocyte nuclear factor 4; VCR, vincristine; siRNA, small interfering RNA.

All patients with GC were followed-up for 70 months $(n=126)$. The Kaplan-Meier estimator survival curves were plotted according to the HNF- $4 \alpha$ level in gastric cancer tissues, and the patients with strong positive (+++) HNF-4 $\alpha$ expression exhibited the poorest survival rate among those three groups (Fig. 5).

\section{Discussion}

On the basis of the high-throughput profiling analysis, several subsets of TFs were suggested to serve a role in drug 


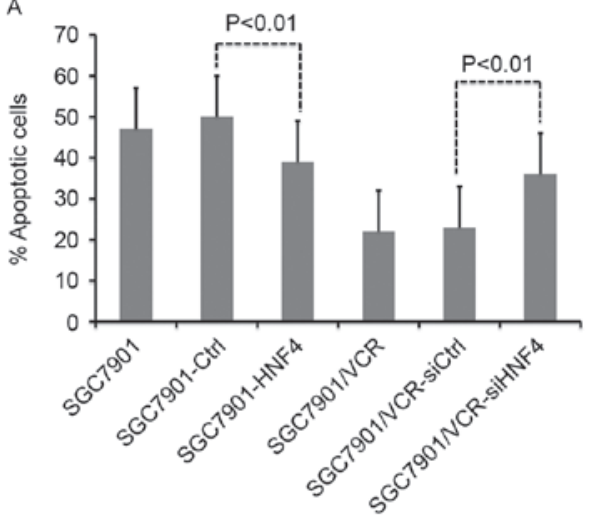

C

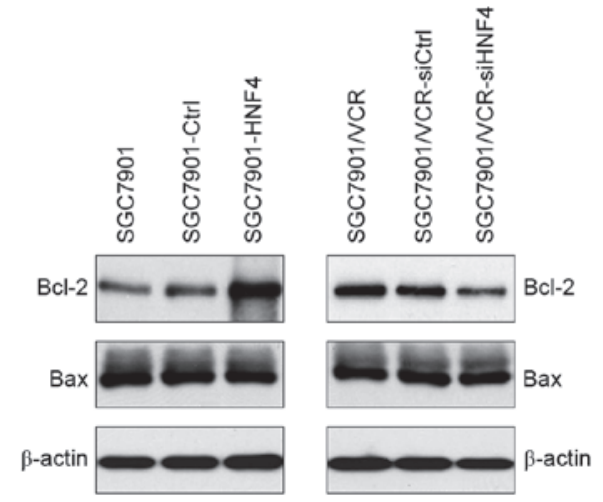

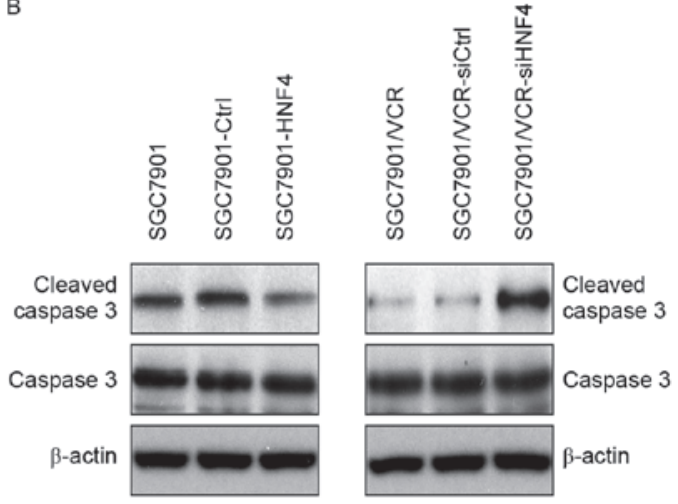

D

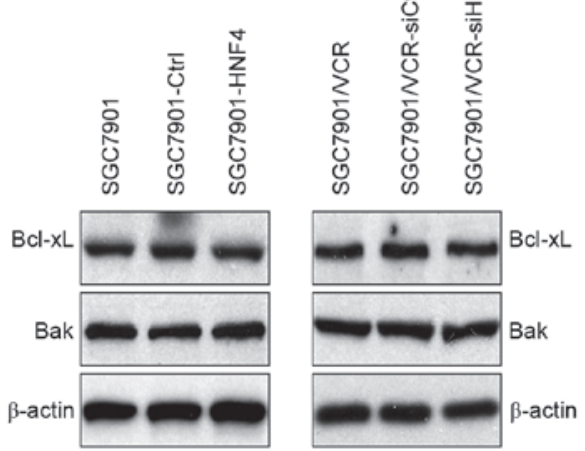

Figure 3. HNF-4 $\alpha$ promotes VCR-induced apoptosis and Bcl-2 expression in gastric cancer cells. (A) Gastric cancer cells and their variants were treated with VCR and cell apoptosis was evaluated using an Annexin V-fluorescein isothiocyanate assay and flow cytometry. (B) The expression of total and cleaved caspase-3 in VCR-treated gastric cancer cells was evaluated using western blotting. (C) The expression of Bcl-2 and Baxin gastric cancer cell variants was detected by western blotting. (D) The expression of Bcl-XL and Bak in gastric cancer cell variants was determined by western blotting. $\beta$-actin served as a loading control. Results are representative of 3 independent experiments. Ctrl, control; HNF4, hepatocyte nuclear factor 4; VCR, vincristine; Bcl-2, B-cell lymphoma 2; Bax, Bcl-associated X protein; Bcl-XL, B-cell lymphoma extra-large.

resistance in cancer. In the present study, TF Activation Profiling Plate Arrays were performed using GC cell models with distinct chemo-sensitivities. Previous studies have identified several TFs associated with drug resistance in GC. For example, it was revealed that HIF-1-dependent pathways were activated in chemo-resistant GC cells, and MGrl-antigen (MGr1-Ag)/37 kDa laminin receptor precursor (37LRP), mitogen-activated protein kinases/extracellular-signal-related kinases and phosphatase and tensin homologue (PTEN)/protein kinase B (Akt) were frequently identified to be synergistically altered with HIF-1 (14-16). NF- $\mathrm{B}$, a key TF in inflammation was also identified to be associated with MDR in GC. Inhibition of NF- $\mathrm{BB}$ activation was able to reverse drug resistance, and pathways including p53 and PTEN/Akt were also involved in NF- $\kappa \mathrm{B}$ activation $(8,17,18)$. In addition, the NF- $\kappa B-S n a i l-B c l-2$ axis was also identified to be associated with mitochondrial antioxidant manganese superoxide dismutase-induced cisplatin resistance in lung cancer (19). These previous studies assist in confirming the reliability of the results of the present study.

HNF- $4 \alpha$ is a member of the orphan steroid hormone nuclear receptor superfamily, and it activates a diverse set of liver genes, including transthyretin and $\alpha_{1}$-antitrypsin in early liver development (20). However, the roles of HNF-4 $\alpha$ in cancer development are not yet understood. According to a recent study, HNF- $4 \alpha$ was demonstrated to be downregulated by hepatitis B viral protein, leading to cytochrome
P450 2E1gene inhibition (21), which suggested the activation of this signaling pathway may contribute to hepatocarcinogenesis (21). Intrahepatic cholangiocarcinoma (IHCC) was associated with the genetic alterations in isocitrate dehydrogenase 1 (IDH1) and IDH2. A recent study identified that IDH mutation was able to block the hepatocyte differentiation of liver progenitor cells by suppressing HNF- $4 \alpha$, indicating a functional role in IDH-driven IHCC pathogenesis (22). In a study by Schwartz et al (23), knockdown of HNF-4 $\alpha$ in colorectal cancer led to a decreased proliferation rate, and inhibited the proliferation in HT22 and Caco 2 cells.

The present study identified that HNF- $4 \alpha$ was also significantly over-activated in chemo-resistant GC cells; however, its exact function in GC was unclear. In the present study, the effects of HNF- $4 \alpha$ on MDR of GC were investigated, and it was demonstrated that the chemo-sensitivities of GC cells maybe significantly affected by HNF-4 $\alpha$. Chemo-resistance was promoted by HNF- $4 \alpha$ through the inhibition of cell apoptosis; however, this phenomenon was not associated with drug transportation. The results of the present study suggest that HNF- $4 \alpha$ may serve an important role in malignant phenotypes of GC. Multiple studies are in agreement with these results $(24,25)$. Kojima et al (24) performed immunohistochemical studies in 35 cases of gastric adenocarcinomas and corresponding non-neoplastic gastric tissues GC. It was demonstrated that in non-neoplastic and neoplastic gastric glandular cells, the expression of HNF- $4 \alpha$ was associated with the intestinal 
A

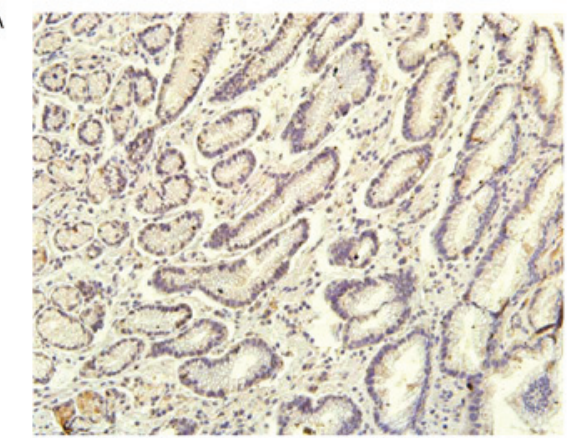

C

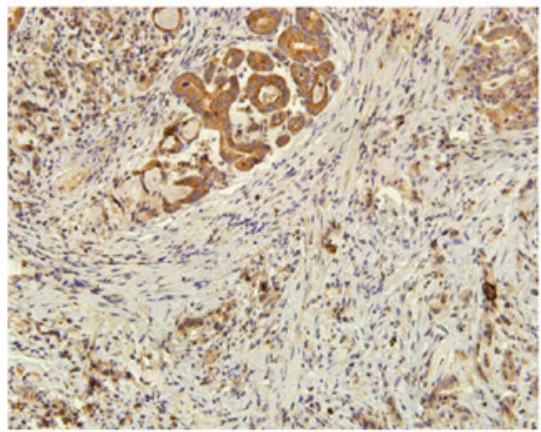

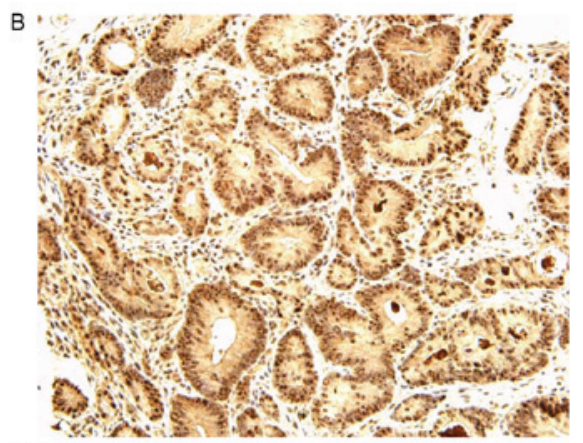

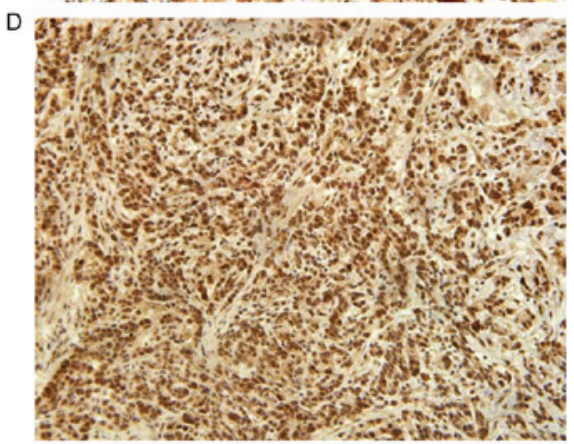

E

\begin{tabular}{|c|c|c|c|c|c|c|}
\hline & \multirow{2}{*}{ Cases } & \multicolumn{4}{|c|}{ HNF- $-4 \alpha$ expression } & \multirow{2}{*}{ - P value } \\
\hline & & - & + & ++ & +++ & \\
\hline Chronic gastritis & 69 & 3 & 47 & 16 & 3 & $<0.01$ \\
\hline Gastric cancer & 126 & 2 & 15 & 48 & 61 & \\
\hline
\end{tabular}

Figure 4. HNF-4 $\alpha$ is enhanced in human gastric cancer tissues. Expression of HNF-4 $\alpha$ was detected using immunohistochemical staining in chronic gastritis and gastric cancer tissues. (A) Chronic gastritis. (B) Well-differentiated gastric cancer. (C) Moderately differentiated gastric cancer. (D) Poorly differentiated gastric cancer. Images are representative of (magnification, x200) 69 cases of chronic gastritis and 39, 45, 42 cases of well-, moderately and poorly differentiated gastric cancer, respectively. (E) The difference between HNF-4 $\alpha$ expression in gastric cancer and chronic gastritis was analyzed using the $\chi^{2}$ test. HNF-4 $\alpha$, hepatocyte nuclear factor $4 \alpha$.

phenotype, which suggested that HNF- $4 \alpha$ may participate in the development and maintenance of the intestinal phenotype of the gastric mucosa and adenocarcinomas. Hepatoid carcinomas, including $\alpha$-fetoprotein-producing gastric carcinoma cells also demonstrated an upregulation in HNF-4 $\alpha$ (25). This phenomenon may be attributed to its function in liver development and its transactivation of liver-associated genes. In the present study, HNF- $4 \alpha$ was markedly expressed in CG, significantly upregulated in GC tissues, and associated with GC tumor stage and differentiation, indicating that HNF- $4 \alpha$ serves a role in gastric carcinogenesis. Furthermore, the HNF- $4 \alpha$ expression level in GC tissues is inversely associated with patient survival. Therefore, future studies evaluating the prognostic value of HNF- $4 \alpha$ for patients with GC are recommended.

The results of the present study indicate the potential for HNF- $4 \alpha$ to regulate MDR of GC; however, its underlying molecular mechanism remains unclear. An important mechanism of HNF- $4 \alpha$ regulation may be epigenetic modulation. The effects of HNF- $4 \alpha$ on cell proliferation have been observed in various colorectal cancer cells, and histone deacetylase inhibitor targeting HNF-4 $\alpha$ were able to suppress proliferation of colon cancer cells (26). Notably, NF- $\kappa B$, HIF-1 and HNF-4 $\alpha$ were the most over-activated TFs in the

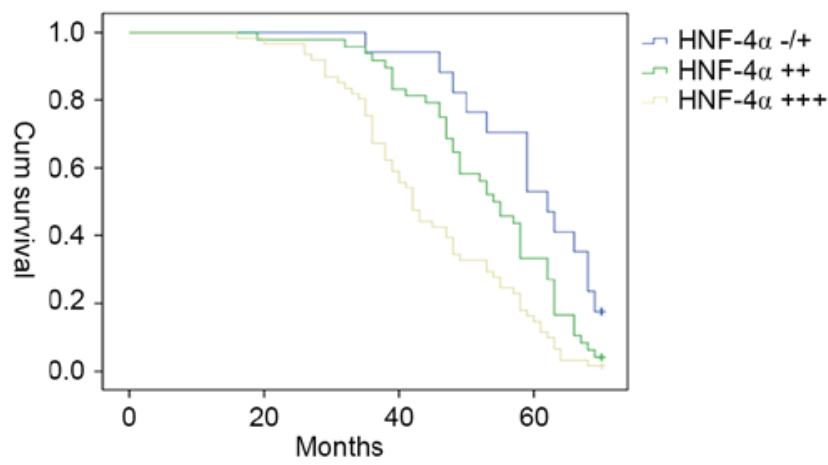

Figure 5. HNF-4 $\alpha$ expression levels are inversely associated with survival of patients with gastric cancer. All patients with gastric cancer $(n=126)$ were monitored for 70 months. These patients were divided into three groups according to the HNF- $4 \alpha$ level as described in Fig. 4. Kaplan-Meier estimator survival curves were created and analyzed using SPSS software. $\mathrm{P}<0.001$ (HNF- $4 \alpha+++$ vs. HNF-4 $\alpha-/+$; HNF- $4 \alpha+++$ vs. HNF- $4 \alpha++$ ) and $\mathrm{P}=0.002$ (HNF- $4 \alpha++$ vs. HNF- $4 \alpha-/+$ ) at the end of follow-up indicated significant difference among the three groups. Cum, cumulative; HNF- $4 \alpha$, hepatocyte nuclear factor $4 \alpha$.

present study, and their mutual interaction may also contribute to the function of HNF- $4 \alpha$. According to a previous study in hepatic cells, tumor necrosis factor- $\alpha$ or other factors (latent 
membrane protein 1 of the Epstein-Barr virus and wild-type forms of NF- $\mathrm{kB}$ signalling mediators) was able to suppress the transcriptional activity of HNF-4-dependent promoters by triggering the NF- $\kappa \mathrm{B}$ response (27). Furthermore, it was shown that this inhibition could be accounted for by a decrease in DNA binding and the downregulation of the transactivation potential of the activation functions 1 and 2 (AF-1 and AF-2) domains of HNF-4 $\alpha$ (27). In primary rat hepatocytes, glucokinase gene expression was associated with HIF-1 and HNF-4 in a PI3K/Akt-dependent signaling pathway (28). Another study demonstrated that the transitional change occurred from the interaction of HIF-1 and HNF-4 under hypoxic conditions (29). Considering the fact that HIF-1 serves important roles in MDR in GC, the cross-talk of HIF-1 and HNF-4 may be the potential mechanism underlying the regulatory function of HNF-4 in MDR. The present study demonstrated that ectopic expression of HNF- $4 \alpha$ was able to upregulate Bcl-2 in $\mathrm{GC}$ cells. This effect was specific for Bcl-2 owing to several other apoptosis-related molecules including Bax, Bak and Bcl-xL not being influenced by HNF- $4 \alpha$. It is reasonable to hypothesize that HNF- $4 \alpha$ promoted MDR of GC cells, at least through regulating $\mathrm{Bcl}-2$ expression.

To conclude, HNF- $4 \alpha$ is a TF that regulates MDR in GC. It inhibits drug-induced apoptosis and promotes GC-related MDR in vitro; however, further studies are required to further elucidate the underlying molecular mechanisms. The results of the present study suggest that HNF- $4 \alpha$ may serve as an important target for MDR management.

\section{References}

1. Siegel RL, Miller KD and Jemal A: Cancer statistics, 2015. CA Cancer J Clin 65: 5-29, 2015.

2. Fodale V, Pierobon M, Liotta L and Petricoin E: Mechanism of cell adaptation: When and how do cancer cells develop chemoresistance? Cancer J 17: 89-95, 2011.

3. Noguchi K, Katayama K and Sugimoto Y: Human ABC transporter ABCG2/BCRP expression in chemoresistance: Basic and clinical perspectives for molecular cancer therapeutics. Pharmgenomics Pers Med 7: 53-64, 2014

4. Elkholi R, Renault TT, Serasinghe MN and Chipuk JE: Putting the pieces together: How is the mitochondrial pathway of apoptosis regulated in cancer and chemotherapy? Cancer Metab 2: 16, 2014.

5. Pflaum J, Schlosser S and Müller M: p53 family and cellular stress responses in cancer. Front Oncol 4: 285, 2014.

6. Amelio I and Melino G: The p53 family and the hypoxia-inducible factors (HIFs): Determinants of cancer progression. Trends Biochem Sci 40: 425-434, 2015.

7. Ozaki T, Nakamura M and Shimozato O: Novel implications of DNA damage response in drug resistance of malignant cancers obtained from the functional interaction between p53 family and RUNX2. Biomolecules 5: 2854-2876, 2015.

8. Endo F, Nishizuka SS, Kume K, Ishida K, Katagiri H, Ishida K, Sato K, Iwaya T, Koeda K and Wakabayashi G: A compensatory role of NF- $\mathrm{\kappa B}$ to $\mathrm{p} 53$ in response to 5-FU-based chemotherapy for gastric cancer cell lines. PLoS One 9: e90155, 2014.

9. Prabhu L, Mundade R, Korc M, Loehrer PJ and Lu T: Critical role of NF- $\kappa B$ in pancreatic cancer. Oncotarget 5: 10969-10975, 2014.

10. Li F, Zhang J, Arfuso F, Chinnathambi A, Zayed ME, Alharbi SA, Kumar AP, Ahn KS and Sethi G: NF-kB in cancer therapy. Arch Toxicol 89: 711-731, 2015.

11. Hong L, Qiao T, Han Y, Han S, Zhang X, Lin T, Gao J, Zhao P, Chen Z and Fan D: ZNRD1 mediates resistance of gastric cancer cells to methotrexate by regulation of IMPDH2 and Bcl-2. Biochem Cell Biol 84: 199-206, 2006.
12. Zhao L, Pan Y, Gang Y, Wang H, Jin H, Tie J, Xia L, Zhang Y, $\mathrm{He} \mathrm{L}$, Yao L, et al: Identification of GAS1 as an epirubicin resistance-related gene in human gastric cancer cells with a partially randomized small interfering RNA library. J Biol Chem 284: 26273-26285, 2009.

13. Su L, Liu X, Chai N, Lv L, Wang R, Li X, Nie Y, Shi Y and Fan D: The transcription factor FOXO4 is down-regulated and inhibits tumor proliferation and metastasis in gastric cancer. BMC Cancer 14: 378, 2014

14. Liu L, Sun L, Zhang H, Li Z, Ning X, Shi Y, Guo C, Han S, Wu K and Fan D. Hypoxia-mediated up-regulation of MGr1-Ag/37LRP in gastric cancers occurs via hypoxia-inducible-factor 1-dependent mechanism and contributes to drug resistance. Int $\mathbf{J}$ Cancer 124: 1707-1715, 2009.

15. Liu L, Zhang H, Sun L, Gao Y, Jin H, Liang S, Wang Y, Dong M, Shi Y, Li Z, et al: ERK/MAPK activation involves hypoxia-induced MGr1-Ag/37LRP expression and contributes to apoptosis resistance in gastric cancer. Int J Cancer 127: 820-829, 2010.

16. Chen F, Zhuang M, Zhong C, Peng J, Wang X, Li J, Chen Z and Huang Y: Baicalein reverses hypoxia-induced 5-FU resistance in gastric cancer AGS cells through suppression of glycolysis and the PTEN/Akt/HIF-1 $\alpha$ signaling pathway. Oncol Rep 33: 457-463, 2015.

17. Zhou W, Fu XQ, Zhang LL, Zhang J, Huang X, Lu XH, Shen L, Liu BN,Liu J,Luo HS, etal:The AKT1/NF-kappaB/Notch1/PTEN axis has an important role in chemoresistance of gastric cancer cells. Cell Death Dis 4: e847, 2013.

18. Zhi X, Tao J, Xiang G, Cao H, Liu Z, Yang K, Lv C and Ni S: APRIL induces cisplatin resistance in gastric cancer cells via activation of the NF- $\kappa$ B pathway. Cell Physiol Biochem 35: 571-585, 2015.

19. Chen PM, Cheng YW, Wu TC, Chen CY and Lee H: MnSOD overexpression confers cisplatin resistance in lung adenocarcinoma via the NF- $\mathrm{B} / \mathrm{S}$ nail/Bcl-2 pathway. Free Radic Biol Med 79: 127-137, 2015.

20. Hayashi $Y$, Wang W, Ninomiya T, Nagano $H$, Ohta $\mathrm{K}$ and Itoh $\mathrm{H}$ : Liver enriched transcription factors and differentiation of hepatocellular carcinoma. Mol Pathol 52: 19-24, 1999.

21. Liu H, Lou G, Li C, Wang X, Cederbaum AI, Gan L and Xie B: $\mathrm{HBx}$ inhibits CYP2E1 gene expression via downregulating HNF4 $\alpha$ in human hepatoma cells. PLoS One 9: e107913, 2014.

22. Saha SK, Parachoniak CA, Ghanta KS, Fitamant J, Ross KN, Najem MS, Gurumurthy S, Akbay EA, Sia D, Cornella H, et al: Mutant IDH inhibits HNF- $4 \alpha$ to block hepatocyte differentiation and promote biliary cancer. Nature 513: 110-114, 2014.

23. Schwartz B, Algamas-Dimantov A, Hertz R, Nataf J, Kerman A, Peri I and Bar-Tana J: Inhibition of colorectal cancer by targeting hepatocyte nuclear factor-4alpha. Int J Cancer 124: 1081-1089, 2009.

24. Kojima K, Kishimoto T, Nagai Y, Tanizawa T, Nakatani Y, Miyazaki $M$ and Ishikura $H$ : The expression of hepatocyte nuclear factor-4alpha, a developmental regulator of visceral endoderm, correlates with the intestinal phenotype of gastric adenocarcinomas. Pathology 38: 548-554, 2006.

25. Supriatna Y, Kishimoto T, Furuya M, Tochigi N, Ishiguro H, Tosh D and Ishikura H: Expression of liver-enriched nuclear factors and their isoforms in alpha-fetoprotein-producing gastric carcinoma cells. Exp Mol Pathol 82: 316-321, 2007.

26. Algamas-Dimantov A, Yehuda-Shnaidman E, Peri I and Schwartz B: Epigenetic control of HNF- $4 \alpha$ in colon carcinoma cells affects MUC4 expression and malignancy. Cell Oncol (Dordr) 36: 155-167, 2013.

27. Nikolaidou-Neokosmidou V, Zannis VI and Kardassis D: Inhibition of hepatocyte nuclear factor 4 transcriptional activity by the nuclear factor kappaB pathway. Biochem J 398: 439-450, 2006.

28. Roth U, Curth K, Unterman TG and Kietzmann T: The transcription factors HIF-1 and HNF-4 and the coactivator p300 are involved in insulin-regulated glucokinase gene expression via the phosphatidylinositol 3-kinase/protein kinase B pathway. J Biol Chem 279: 2623-2631, 2004.

29. Zhang W, Tsuchiya T and Yasukochi Y: Transitional change in interaction between HIF-1 and HNF-4 in response to hypoxia. J Hum Genet 44: 293-299, 1999. 